\title{
Transient development of capillary-gravity waves in a running stream
}

\section{Kalyan Kumar Bagchi and Lokenath Debnath}

\begin{abstract}
An initial value investigation is made of the propagation of capillary-gravity waves generated by an oscillating pressure distribution acting at the free surface of a running stream of finite, infinite, and shallow depth. The solution for the free surface elevation is obtained explicitly by using the generalized Fourier transform and its asymptotic expansion. It is found that the solution consists of both the steady state and the transient components. The latter decays asymptotically as $t \rightarrow \infty$ and the ultimate steady state is attained. It is shown that the steady state consists of two or four progressive capillary-gravity waves travelling both upstream and downstream according as the basic stream velocity is less or greater than the critical speed. Special attention is given to the existence of the critical values associated with the running stream of finite, infinite, and shallow depth. A comparison is made between the unsteady wave motions in an inviscid fluid with or without surface tension.
\end{abstract}

Received $3 \mathrm{July}$ 1973. The first author is grateful to the Centre of Advanced Studies in Applied Mathematics, University of Calcutta, for a Research Fellowship. The second author expresses his sincere thanks to the Centre for a Visiting Professorship. Both express their heartiest thanks to Dr M. Dutta and Professor P.K. Ghosh of the Centre for their encouragement and interest in the work. This work was partly supported by the Research Council of East Carolina University. 


\section{Introduction}

Recently, Debnath and Rosenblat [2] have made an initial value investigation of the propagation of two-dimensional waves generated by an oscillating. pressure distribution acting at the free surface of an inviscid running stream of finite depth. With the aid of the generalized function method and asymptotic devices, they have solved the wave problem and determined the nature of the unsteady wave motions. It has been shown that the ultimate steady state consists of either two or four waves depending on the relative magnitudes of the velocity of the stream, its depth, and the forcing frequency of the applied pressure. Special attention is given to the singular behavior of the free surface elevation on the critical curve separating these two possible states of the solution. Several limiting cases of interest have been discussed.

In recent years, interest in the linearized theory of water waves including the effects of surface tension has been revived by Evans [3, 4], Rhodes-Robinson [9], Packham [8], and Debnath [1]. Most of these works are concerned with the propagation of surface waves due to submerged sources, singularities, or obstacles of localised disturbances applied at the free surface of the liquid. In spite of these works, an attempt has hardly been made to study the development of surface waves generated by disturbances acting at the free surface of a running stream which includes the effect of surface tension.

The present analysis is intended to make a study of the transient development of capillary-gravity waves generated by an oscillatory pressure acting at the free surface of a running stream of finite, infinite, and shallow depth. With the aid of the generalized Fourier transform and its asymptotic expansion, the solution for the free surface displacement is found explicitly. It is shown that the solution consists of both the steady state and the transient components. The transient effects decay asymptotically as $t \rightarrow \infty$ and the ultimate steady state is reached. It is predicted that the steady state consists of two or four progressive capillary-gravity waves travelling both upstream and downstream according as the stream velocity is less or greater than the critical speed. Special emphasis is given to the existence of the critical values related to the running stream of finite, infinite, and shallow depth. A comparison is made 
between the unsteady wave motions in an inviscid fluid with or without surface tension.

\section{Mathematical formulation}

Based upon the assumptions of the linearized theory of water waves, we consider the propagation of capillary-gravity waves on the surface of an inviscid, incompressible, homogeneous liquid including the effects of surface tension. The unsteady analysis is carried out under the following assumptions :

(i) in an undisturbed state, an infinitely horizontal extent of liquid has constant depth $H$ and flows with uniform velocity $U_{0}$;

(ii) the liquid has constant density $\rho$ and surface tension $T_{1}^{*}$;

(iii) the wave-generating mechanism is a harmonically oscillating pressure applied at the free surface of the liquid;

(iv) the applied pressure distribution is two-dimensional so that the wave motion is entirely parallel to the flow.

It is convenient to take the cartesian coordinates so that the origin is situated on the undisturbed free surface, the $z$-axis is vertical positive upward and the basic stream moves parallel to the $X$-axis with the velocity $U_{0}$ relative to the coordinate frame.

The applied pressure distribution may be an arbitrary function of $X$ but it would be enough for the investigation of the principal features of the unsteady flow to take the simple form

$$
p(X, T)=P_{0} \delta(X) e^{i \omega T_{H}(T)},
$$

where $P_{0}$ is constant, $\delta(X)$ is the Dirac delta function and $H(T)$ is the Heaviside unit function of time $T$.

As the motion is irrotational, there exists a disturbance velocity potential $\Phi(X, 2 ; T)$ which satisfies the Laplace equation 


$$
\nabla^{2} \Phi \equiv \frac{\partial^{2} \Phi}{\partial X^{2}}+\frac{\partial^{2} \Phi}{\partial z^{2}}=0
$$

in $-H \leq Z \leq 0,-\infty<X<\infty, T>0$.

Taking into account the effect of the surface tension, the linearized free surface conditions are

$$
\begin{gathered}
\frac{\partial \Phi}{\partial T}+U_{0} \frac{\partial \Phi}{\partial X}+g E-\frac{T_{1}^{*}}{\rho} \frac{\partial^{2} E}{\partial X^{2}}=-p(X, T), \quad Z=0, T>0, \\
\frac{\partial E}{\partial T}+U_{0} \frac{\partial E}{\partial X}=\frac{\partial \Phi}{\partial Z}, Z=0, T>0,
\end{gathered}
$$

where $E=E(X, T)$ denotes the vertical surface elevation and $g$ is the acceleration due to gravity.

The boundary condition at the bottom is

$$
\frac{\partial \Phi}{\partial Z}=0 \quad \text { on } \quad Z=-H, T>0
$$

The initial conditions of the problem are

$$
\Phi(X, Z ; 0)=E(X, 0)=0 .
$$

Further, we shall treat $\Phi$ and $E$ as the generalized functions of $X$ in the sense of Lighthill [7] so that their Fourier transforms with respect to $X$ exist.

\section{The solution of the initial value problem}

It is convenient to introduce the dimensionless quantities defined by

$$
\begin{gathered}
(x, z, h)=\frac{\omega^{2}}{g}(X, z, H), \quad t=\omega r, \phi=\frac{P_{0} \omega^{5}}{\rho g^{4}} \Phi, \eta=\frac{P_{0} \omega^{4}}{\rho g^{3}} E, \\
P=\left(\frac{P_{0} \omega^{2}}{\rho g^{2}}\right)^{2}, \quad U=\frac{\omega}{g} U_{0} \text { and } T_{1}=\frac{T_{1}^{*} \omega^{4}}{\rho g^{3}} .
\end{gathered}
$$

It is then easy to rewrite the equations (2.2)-(2.6) in terms of these non-dimensional quantities. In order to solve the initial value problem, we take the Fourier transform with respect to $x$ defined by $[7,10]$, 


$$
\bar{\phi}(k, z ; t)=\frac{1}{\sqrt{2 \pi}} \int_{-\infty}^{\infty} e^{-i k x} \phi(x, z ; t) d x .
$$

Thus the solution of the transformed non-dimensional equation with the bottom boundary condition is

$$
\bar{\phi}(k, z ; t)=\bar{A}(k, t) \cosh k(z+h),
$$

where $\bar{A}$ and $\bar{\eta}$ are determined from the transformed non-dimensional free surface conditions

$$
\begin{aligned}
\frac{d \bar{\phi}}{d t}+U i k \bar{\phi}+ & \left(1+T_{1} k^{2}\right) \bar{n} & =-\frac{P}{\sqrt{2 \pi}} e^{i t}, & z=0, t>0, \\
\frac{d \bar{n}}{d t}+U i k \bar{n} & =\frac{d \bar{\phi}}{d z}, & & z=0, t>0,
\end{aligned}
$$

with the initial conditions

$$
\bar{\phi}(k, z ; 0)=\bar{\eta}(k, 0)=0 \text {. }
$$

The solution for $\bar{n}(k, t)$ can readily be obtained from the above as

$$
\bar{n}(k, t)=\frac{P}{2 \sqrt{2 \pi}}\left(\frac{k \tanh k h}{1+T_{1} k^{2}}\right)^{\frac{1}{2}}\left[\frac{e^{i t}-e^{i m_{1} t}}{1-m_{1}}-\frac{e^{i t}-e^{i m_{2} t}}{1-m_{2}}\right],
$$

where $m_{1}$ and $m_{2}$ are given by

$$
m_{1}, m_{2}=-k U \pm\left\{\left(1+T_{1} k^{2}\right) k \tanh k h\right\}^{\frac{3}{2}} .
$$

The inverse Fourier transformation gives the integral solution for $n(x, t)$ as

$$
n(x, t)=\frac{P}{4 \pi} \int_{-\infty}^{\infty}\left(\frac{k \tanh k h}{1+T_{1} k^{2}}\right)^{\frac{3}{2}}\left[\frac{e^{i t}-e^{i m_{1} t}}{1-m_{1}}-\frac{e^{i t}-e^{i m_{2} t}}{1-m_{2}}\right] e^{i k x} d k .
$$

In the case of infinite depth, $h \rightarrow \infty$ so that $|k| \tanh k h \sim|k|$ and (3.8) reduces to

$$
\eta(x, t)=\frac{P}{4 \pi} \int_{-\infty}^{\infty}\left(\frac{|k|}{1+T_{1} k^{2}}\right)^{\frac{1}{2}}\left[\frac{e^{i t}-e^{i m_{1} t}}{1-m_{1}}-\frac{e^{i t}-e^{i m_{2} t}}{1-m_{2}}\right] e^{i k x} d k,
$$


where $m_{1}$ and $m_{2}$ are to be replaced by their limiting values $-k U \pm\left\{\left(1+T_{1} k^{2}\right\}|k|\right\}^{\frac{2}{2}}$

Similarly; the integral solution for the wave potential $\phi(x, z ; t)$ can be found in both finite and infinite depth cases.

It is noted that the principal features of the unsteady wave motions can be determined completely if integrals (3.8)-(3.9) are evaluated exactly or asymptotically. It should be acknowledged that the exact evaluation of (3.8)-(3.9) is a formidable task. It is thus necessary to resort to asymptotic methods. We next turn our attention to the asymptotic analysis.

\section{Asymptotic analysis}

In order to carry out the asymptotic analysis, it is convenient to rewrite $(3.8)$ as

$$
n(x, t)=\frac{P}{4 \pi}\left[e^{i t} I-J\right]
$$

where $I$ and $J$ are

$$
I=\int_{-\infty}^{\infty}\left(\frac{k \tanh k h}{1+T_{1} k^{2}}\right)^{\frac{1}{2}}\left(\frac{1}{1-m_{1}}-\frac{1}{1-m_{2}}\right) e^{i k x} d x
$$

$$
J=\int_{-\infty}^{\infty}\left(\frac{k \tanh k h}{1+T_{1} k^{2}}\right)^{\frac{2}{2}}\left(\frac{e^{i m_{1} t}}{m_{1}-1}-\frac{e^{i m_{2} t}}{m_{2}-1}\right) e^{i k x} d k
$$

To evaluate these wave integrals for large values of $|x|$ and $t$, we shall use the formulae advanced by Lighthill [7] and Jones [5] for the asymptotic expansion of Fourier transforms of generalized functions. The nature of the ultimate wave system and of the transients is determined by the singularities and stationary points of the wave integrals (4.2)-(4.3). We next turn our attention to the nature and location of these singularities and stationary points.

The significant contribution to $I$, as $|x| \rightarrow \infty$, comes from the poles of the integrand in (4.2), that is, from the roots of the equations 


$$
(4.4 \mathrm{a}, \mathrm{b}) \quad 1+k U= \pm\left\{\left(1+T{ }_{1} k^{2}\right) k \tanh k h\right\}^{\frac{3}{2}} \text {. }
$$

These poles are determined from the points of intersections of the curve $\left\{\left(1+T_{1} k^{2}\right) k \tanh k h\right\}^{\frac{1}{2}}$ with the straight lines $\pm(1+k U)$ for different values of the parameters $U, \omega$ and $h$; and are shown in Figures 1 and 2 . These figures give one positive pole at $k=s_{2}$ and one negative pole at $s=-s_{1}$, provided Figure 1 is relevant. On the other hand, whenever $U>U_{c}^{*}$, there are two further poles arising from Figure 2 at the negative values of $k$ given by $k=-\sigma_{1}$ and $k=-\sigma_{2} ;$ where $U_{c}^{*}$ is the critical value of $U$ whose details will be examined later on.

Following the procedure advanced by Debnath and Rosenblat [2], the steady state wave integral (4.2) can be directly evaluated to obtain (4.5) $I \sim \pi i \operatorname{sgn} x\left[\phi\left(-s_{1}\right) e^{-i s_{1} x}+\phi\left(s_{2}\right) e^{i s_{2} x}\right.$

$$
\left.-H\left(U-U_{c}\right)\left\{\psi\left(-\sigma_{1}\right) e^{-i \sigma_{1} x}+\psi\left(-\sigma_{2}\right) e^{-i \sigma_{2} x}\right\}\right],
$$

where

$(4.6 \mathrm{a}, \mathrm{b}) \quad \phi(k)=-\frac{\left\{k \tanh k h / 1+T{ }_{1} k^{2}\right\}^{\frac{1}{2}}}{\left(d m_{1} / d m\right)}, \quad \psi(k)=-\frac{\left\{k \tanh k h / 1+T{ }^{2} k^{2}\right\}^{\frac{3}{2}}}{\left(d m_{2} / d m\right)}$.

The method of stationary phase [Jones, 5] for the generalized Fourier integral can be employed for the asymptotic evaluation of $J$ for large $t$.

For $|x| \ll U t$, the stationary points of the integral (4.2) are the solutions of the equations
$(4.7 a, b)$
$\frac{d}{d k} f(k)= \pm U$

where

$$
f(k)=\left\{\left(1+r_{1} k^{2}\right) k \tanh k h\right\}^{\frac{2}{2}} .
$$

Unlike in the case without surface tension $f^{\prime}(k)$ is not a monotonic 


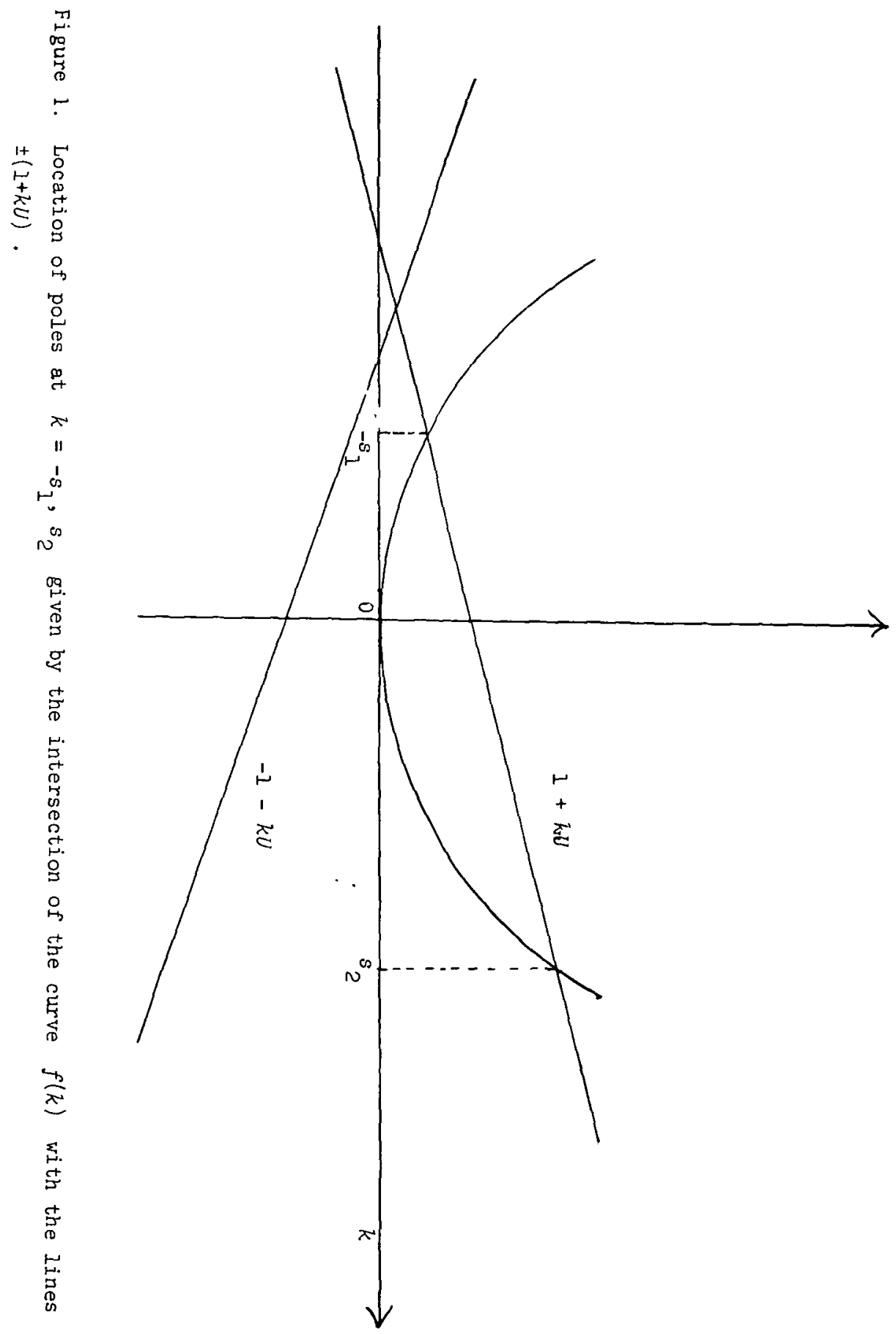




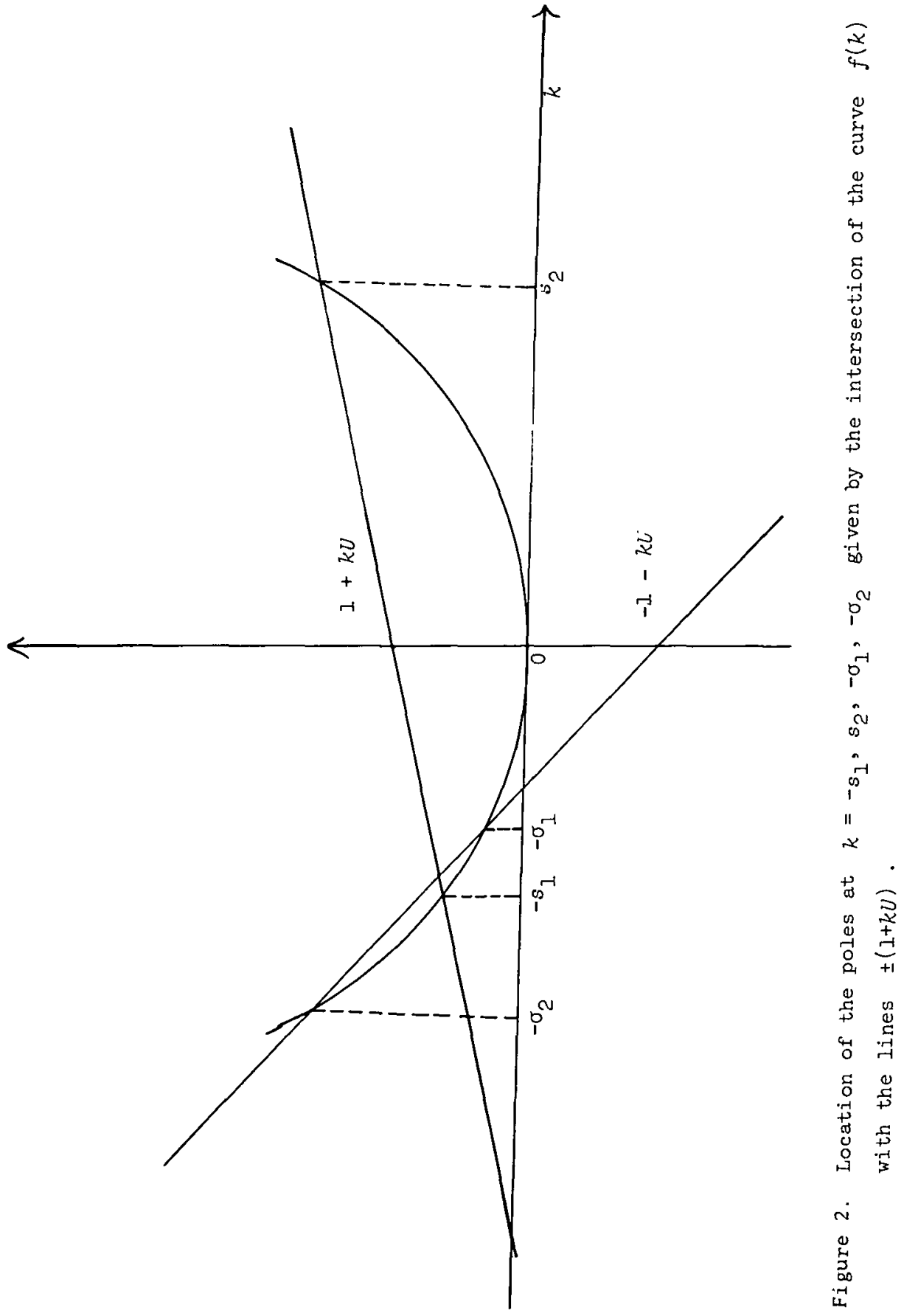


function of $k$. It appears from the graphs of $f(k)$ that $f^{\prime}(k)$ starts from $\sqrt{h}$ at $k=0$ and decreases monotonically to attain a minimum at $k=k_{0}$ and then increases monotonically without any limit as $k \rightarrow \infty$, where $k_{0}$ is the root of the equation $f^{\prime \prime}(k)=0$. Thus a necessary and sufficient condition for the existence of roots of the equations $(4.7 \mathrm{a}, \mathrm{b})$ is that $U>k_{0}$. Hence each of these equations has two roots in the range $f^{\prime}\left(k_{0}\right)<U<\sqrt{h}$ and has one root whenever $U>\sqrt{h}$.

In the case of infinite depth, $f(k)=\left(1+T_{1} k^{2}\right)^{\frac{1}{2}}|k|^{\frac{3}{2}}$ so that

$$
f^{\prime}(k)=\frac{1+3 T_{1} k^{2}}{2\left\{\left(1+T_{1} k^{2}\right) k\right\}^{\frac{3}{2}}} \text { and } f^{\prime \prime}(k)=\frac{1}{2} \frac{3\left(1+T_{1} k^{2}\right)^{2}-4}{\left\{\left(1+T_{1} k^{2}\right) k\right\}^{3 / 2}} \text { for } k>0 \text {. }
$$

It follows from a careful inspection that $f^{\prime}(k)$ tends to infinity as $k \rightarrow 0^{+}$and $k \rightarrow \infty$; and decreases monotonically from $\infty$ to $f^{\prime}\left(k_{0}\right)$ as $k$ increases from 0 to $k_{0}$. Further, it increases monotonically from $f^{\prime}\left(k_{0}\right)$ to $\infty$ as $k$ goes from $k_{0}$ to infinity. Consequently, each of the equations $f^{\prime}(k)= \pm U$ has two roots when $U>f^{\prime}\left(k_{0}\right)$ where $k_{0}$ is numerically evaluated and is approximately equal to $\left(\frac{0.155}{T_{I}}\right)^{\frac{1}{2}}$. Thus for the fluid of finite depth, there are four stationary points at $k= \pm \sigma, \pm \rho$ when $f^{\prime}\left(k_{0}\right)<U<\sqrt{h}$; and there are two stationary points when $U \geq \sqrt{h}$ and also when $U=f^{\prime}\left(k_{0}\right)$. In the case of infinite depth, there are also four stationary point; at $k= \pm \sigma, \pm p$ provided $U>f^{\prime}\left(k_{0}\right)$ and two stationary points wnenever $U=f^{\prime}\left(k_{0}\right)$. It appears that $U=f^{\prime}\left(k_{0}\right)$ represents another critical velocity.

Using the standard formula for the stationary phase approximation (Jones, [5], p. 323), the transient component of $J$, that is, the dominant contribution to $J$ from the stationary points involved in various cases can be obtained as 
(4.9) $J_{\text {transient }} \sim\left[H\left\{U-f^{\prime}\left(k_{0}\right)\right\}-H(U-\sqrt{h})\right]$

$$
\begin{aligned}
& e^{-\frac{i \pi}{4}}\left[( \frac { \sigma \operatorname { t a n h } \sigma h } { 1 + T _ { 1 } \sigma ^ { 2 } } ) ^ { \frac { 1 } { 2 } } \left\{\left(+\frac{2 \pi}{t \mid f^{\prime \prime}(\sigma) T}\right)^{\frac{1}{2}} \frac{\exp \left[i\left\{t m_{1}(\sigma)+\sigma x\right\}\right]}{1-m_{1}(\sigma)}\right.\right. \\
& \left.-\left(+\frac{2 \pi}{t \mid f^{\prime \prime}(-\sigma)}\right)^{\frac{3}{2}} \frac{\exp \left[i\left\{t m_{2}(-\sigma)-\sigma x\right\}\right]}{1-m_{2}(-\sigma)}\right\} \\
& +\left(\frac{\rho \tanh \rho h}{1+T_{1} \rho^{2}}\right)^{\frac{3}{2}}\left\{\left(+\frac{2 \pi}{t \mid f^{\prime \prime}(\rho)}\right)\right\}^{\frac{3}{2}} \frac{\exp \left[i\left\{t m_{1}(\rho)+\rho x\right\}\right]}{1-m_{1}(\rho)} \\
& \left.-\left(+\frac{2 \pi}{t\left|f^{\prime \prime}(-\rho)\right|}\right)^{\frac{1}{2}} \frac{\exp \left[i\left\{t m_{1}(-\rho)-\rho x\right\}\right]}{1-m_{2}(-\rho)}\right]+o\left(\frac{1}{t}\right) \text {. }
\end{aligned}
$$

This result incorporates all the cases of four, two or no stationary points as indicated earlier. However, the transient solution breaks down at the critical speed $U=f^{\prime}\left(k_{0}\right)$. Hence this case needs further investigation.

It remains to calculate the contribution to $J$ from its doles. Which are the same as the poles of $I$. A procedure similar to that of Debnath and Rosenblat [2] can be employed for finding the polar contribution to $J$.

It may be helpful to write down (4.2) in the form

$$
J=J_{1}-J_{2} \text {, }
$$

so that

$$
J_{1}=\left[\int_{-\infty}^{0}+\int_{\rho}^{\infty}\right]\left(\frac{k \tanh k h}{1+T_{1} k^{2}}\right)^{\frac{1}{2}} \frac{e^{i\left(m_{1} t+k x\right)} d k}{\left(m_{1}-1\right)},
$$

and

$$
J_{2}=H\left(U-U_{c}^{*}\right)\left[\int_{-\infty}^{\rho}+\int_{-\rho}^{\rho}\right]\left(\frac{k \tanh k h}{1+T_{1} k^{2}}\right)^{\frac{1}{2}} \frac{e^{i\left(m_{2} t+k x\right)} d k}{\left(m_{2}-1\right)}
$$

or 
(4.13)

$$
J_{2}=H\left(U-U_{c}^{*}\right)\left[\int_{-\infty}^{-\rho}+\int_{-\rho}^{-\sigma}\right]\left(\frac{k \tanh k h}{1+T_{1} k^{2}}\right)^{\frac{3}{2}} \frac{e^{i\left(m_{2} t+k x\right)} d k}{\left(m_{2}-1\right)},
$$

according as $f^{\prime}(k)=-U$ has one or two roots and $m_{2}( \pm k) \rightarrow-\infty$ as $k+\infty$. We then change the variable of integration from $k$ to $m_{1}=m_{1}(k)$ in integral (4.11), and from $k$ to $m_{2}=m_{2}(k)$ in (4.12)-(4.13). It turns out that

(4.14) $J_{1}=\left[\int_{-\infty}^{0}+\int_{-M_{1}}^{\infty}\right]\left[\left\{\phi(k) e^{i k x}\right\}_{k=k^{-1}\left(m_{1}\right)} /\left(m_{1}-1\right)\right] e^{i m_{1} t} d m_{1}$, which is, invoking result (24) of Debnath and Rosenblat [2],

$$
\sim \pi i e^{i t}\left[-\phi\left(-s_{1}\right) e^{-i s_{1} x}+\phi\left(s_{2}\right) e^{i s_{2} x}\right] \text {, as } t \rightarrow \infty,
$$

where $m_{1}(\rho)=-M_{1}<0$.

Similarly, (4.12)-(4.13) reduce to

(4.16) $J_{2}=H\left(U-U_{c}^{*}\right)\left[\int_{-\infty}^{M_{1}}+\int_{M_{1}}^{0}\right]\left[\left\{\psi(k) e^{i k x}\right\}_{k=k^{-1}\left(m_{2}\right)} /\left(m_{2}-1\right)\right] e^{i m_{2} t} d m_{2}$,

(4.17) $J_{2}=H\left(U-U_{c}^{*}\right)\left[\int_{-\infty}^{M_{1}}+\int_{M_{1}}^{-M_{2}}\right]\left[\left\{\psi(k) e^{i k x}\right\}_{k=k^{-1}\left(m_{2}\right)} /\left(m_{2}-1\right)\right] e^{i m_{2} t} d m_{2}$,

where $m_{1}(-\rho)=M_{1}>0$ and $m_{2}(-\sigma)=-M_{2}<0$ with $\sigma<\rho$.

It follows from formula (24) of Debnath and Rosenblat [2] that

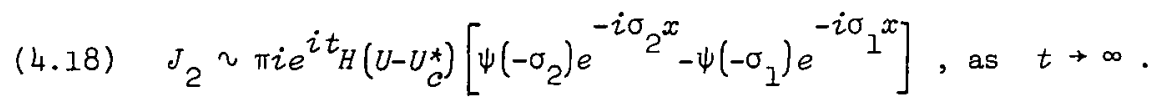

The polar contribution to $J$ as $t \rightarrow \infty$ is therefore given by

(4.19) $J_{\text {polar }} \sim \pi i e^{i t}\left[-\phi\left(-s_{1}\right) e^{-i s_{1} x}+\phi\left(s_{2}\right) e^{i s_{2} x}\right.$

$$
\left.+H\left(U-U_{c}^{*}\right)\left\{\psi\left(-\sigma_{1}\right) e^{-i \sigma_{1} x}-\psi\left(-\sigma_{2}\right) e^{-i \sigma_{2} x}\right\}\right] .
$$


Thus the asymptotic representation of the surface elevation is explicitly obtained as $t \rightarrow \infty$ and can be written as a convenient form (4.20) $n(x, t)=\eta_{s t}(x, t)+\eta_{t r}(x, t)$,

where $\eta_{s t}(x, t)$ is the steady state component and $\eta_{t r}(x, t)$ is the transient solution.

It is noted that the former is essentially made up of the polar contributions to $I$ and $J$ which are given by (4.5) and (4.19) respectively, and has the final representation

(4.21) $\eta_{s t}(x, t) \sim \frac{i P}{2}\left[\phi\left(-s_{1}\right) e^{i\left(t-s_{1} x\right)}-H\left(U-U_{c}^{*}\right) \psi\left(-\sigma_{1}\right) e^{i\left(t-\sigma_{1} x\right)}\right], x>0$, and

$$
\eta_{s t}(x, t) \sim \frac{i P}{2}\left[-\phi\left(s_{2}\right) e^{i\left(t+s_{2} x\right)}+H\left(U-U_{c}^{*}\right) \psi\left(-\sigma_{2}\right) e^{i\left(t-\sigma_{2} x\right)}\right], x<0 .
$$

The significant contribution to the transient solution $\eta_{t r}$ is given by (4.9) which eventually decays in the limit $t \rightarrow \infty$. Thus the steady state is attained in the limit and described by (4.21)-(4.22). Further, the steady state wave system has the following characteristic features:

(a) when $U<U_{c}^{*}$, solutions (4.21)-(4.22) confirm the existence of two progressive capillary-gravity waves on the surface of the running stream. One of these waves is travelling downstream of the origin with the phase velocity $\frac{g}{w s_{1}}$ and the other is propagating upstream with the phase velocity $\frac{g}{w s_{2}}$;

(b) when $U>U_{c}^{*}$, there are four capillary-gravity waves propagating on both upstream and downstream sides of the origin. Three of these waves are travelling downstream with the phase velocities $\frac{g}{\omega s_{1}}, \frac{g}{\omega \sigma}$ and $\frac{g}{\omega \sigma_{2}}$ respectively, and the remaining one is propagating upstream with the speed $\frac{g}{w_{2}}$. 
As it has been found earlier that the nature of the singularities and the stationary points of the wave-integrals involved in (3.9) is somewhat similar to those of (3.8), the conclusions are also true in an infinitely deep fluid with the exceptions of the magnitudes of the phase velocities of these waves.

The present analysis reveals that the structure of the wave system above or below the critical velocity shows a striking contrast to that predicted by Debnath and Rosenblat in an inviscid fluid without surface tension.

\section{The critical values}

It is now necessary to recognize the critical speeds associated with the problem. The critical value occurs when there exists a common root of the equations $(4.4 \mathrm{~b})$ and $(4.7 \mathrm{~b})$, that is,

$$
1+k U+\left\{\left(1+T_{1} k^{2}\right) k \tanh k h\right\}^{\frac{1}{2}}=0
$$

(5.2) $\left\{\left(1+3 T_{1} k^{2}\right) \tanh k h+\left(1+T_{1} k^{2}\right) k h \operatorname{sech}^{2} k h\right\}=-2 U\left\{\left(1+T_{1} k^{2}\right)^{\frac{1}{2}} k \tanh k h\right\}^{\frac{1}{2}}$.

Replacing $k$ by $-\lambda$ and eliminating the hyperbolic function from the above equations, the critical velocity is given by the solution of the equation

$$
\text { (5.3) } \quad\left(1+3 T_{1} \lambda^{2}\right)(1-\lambda U)^{2}+h\left\{\lambda^{2}\left(1+T_{1} \lambda^{2}\right)-(1-\lambda U)^{4}\right\}+2 \lambda U\left(1+T_{1} \lambda^{2}\right)(1-\lambda U)=0 \text {. }
$$

In particular, for an infinitely deep fluid $(h+\infty)$, the critical speed $U_{c}^{*}$ is given by the equation

$$
\text { (5.4) } 4 \omega U^{5}-U^{4}+4 T_{1} \omega^{3} U^{3}+30 T_{1} \omega^{2} U^{2}-24 \omega T_{1} U+4 T_{1}+27 T_{1}^{2} \omega^{4}=0 \text {. }
$$

This result is believed to be new, and when $T_{I} \equiv 0$ with $\omega \neq 0$, the critical value is given by, in dimensional form,

$$
U_{c}^{*} \equiv U_{c}=\frac{g}{4 \omega} .
$$

This result has been independently predicted by Kaplan [6], and Debnath and Rosenb lat [2]. 
Further, when $\omega=0$ with $T_{1} \neq 0$, there exists a new critical speed which has the dimensional form

$$
U_{c}^{*}=\left(\frac{4 g T_{1}^{*}}{\rho}\right)^{\frac{1}{4}}
$$

which depends on the ratio $\left(\frac{T_{1}^{*}}{\rho}\right)$ for a given $g$, and is exactly identical with the minimum velocity of the capillary-gravity waves in deep water without any basic stream.

If we treat equation $(5.4)$ as a quadratic in $T_{1}$, then the existence of at least one positive value of $T_{1}$ requires $U<\frac{1}{4 \omega}$, which is a necessary restriction on the velocity of the basic stream.

In the case of shallow depth, the long wave approximation holds good, so that the equation determining the critical speed has the form

$$
\text { (5.7) }\left\{\frac{1}{12}\left(h-U^{2}\right)-\omega^{2} h T_{1}\right\}^{3}=27\left[\frac{h}{6} T_{1} \omega^{2}\left(h-U^{2}\right)+\frac{h}{4} T_{1} \omega^{2} U^{4}+\left(\frac{h-U^{2}}{6}\right)^{3}\right]^{2} \text {. }
$$

In particular, when $\omega=0$, the dimensional form of the critical velocity is $\sqrt{g H}$ which is obviously unaffected by surface tension.

On the other hand, when surface tension is neglected, equation (5.7) admits no solution. Of course, in this case, the relevant equation for the common root is $k^{2}\left(U^{2}-h\right)+2 k U+1=0$, which has no double root. Thus there is no critical speed in a shallow fluid when its free surface is disturbed by a periodic pressure of frequency $\omega$.

\section{Concluding remarks}

The initial value problem has been solved for the propagation of capillary-gravity waves in an inviscid fluid with surface tension. In the absence of surface tension, the findings of this paper are in excellent agreement with those of Debnath and Rosenblat [2], and of Stoker [11] when the additional restrictions $T_{1}=0, \omega=0$ are made. In real applications, it would be interesting to solve the problem including the viscous effects. Such a study may be made in a subsequent paper. 


\section{References}

[1] L. Debnath, "Effect of surface tension on time dependent gravity waves", Math. Japon. 16 (1971), 81-90.

[2] L. Debnath and S. Rosenblat, "The ultimate approach to the steady state in the generation of waves on a running stream", Quart. J. Mech. App Z. Math. 22 (1969), 221-233.

[3] D.V. Evans, "The influence of surface tension on the reflection of water waves by a plane vertical barrier", Proc. Cambridge Philos. Soc. 64 (1968), 795-810.

[4] D.V. Evans, "The effect of surface tension on the waves produced by a heaving circular cylinder", Proc. Cambridge Phizos. Soc. 64 (1968), 833-847.

[5] D.S. Jones, Generalised functions (McGraw-Hill, New York; Toronto, Ontario; London; 1966).

[6] Paul Kaplan, "The waves generated by the forward motion of oscillatory pressure distributions", Proc. Fifth Midwestem Conf. Fluid Mechanics, 1957, 316-329 (University of Michigan Press, Ann Arbor, Michigan, 1957).

[7] M.J. Lighthill, An introduction to Fourier analysis and generalized functions (Cambridge University Press, Cambridge, 1958).

[8] B.A. Packham, "Capillary-gravity waves against a vertical cliff", Proc. Cambridge Phizos. Soc. 64 (1968), 827-832.

[9] P.F. Rhodes-Robinson, "Fundamental singularities in the theory of water waves with surface tension", Buzz. Austral. Math. Soc. 2 $(1970), 317-333$.

[10] I.N. Sneddon, The use of integral transforms (McGraw-Hill, New York; Toronto, Ontario; London; 1972).

[11] J.J. Stoker, water waves: The mathematical theory with applications (Pure and Applied Mathematics, 4. Interscience, New York, London, 1957).

Centre of Advanced Study

in Applied Mathematics, University of Calcutta, Calcutta, India.
Department of Mathematics, East Carolina University, Greenville, North Carolina, USA. 